\title{
Studies on dilute solution of rodlike macroions: Part 4 Aggregation with enhanced interchain orientational correlation
}

\author{
R. Furukawa and G. C. Berry \\ Chemistry Department, Carnegie-Mellon University, Pittsburgh, PA 15213, USA
}

\begin{abstract}
Polarized and depolarized absolute intensity light scattering, photon correlation light scattering and viscometry studies are reported on solutions in methane sulfonic acid, MSA, poly(1,4-phenylene-2,6-benzobisthiazole), PBT, and copolymers of PBT containing a bipyridyl moiety in place of some of the phenylene residues. It is found that addition of the potassium salt of MSA to the solutions reduces the second virial coefficient and causes pronounced increase in the depolarized scattering, with accompanying changes in other properties studied. The photon correlation light scattering is analyzed in terms of pseudo-components comprising aggregates with substantial orientational correlation among the rodlike chain elements. This behavior may be in accord with predicted behavior for the phase equilibria for rodlike chains.
\end{abstract}

\section{INTRODUCTION}

Light scattering studies (ref. 1-3) on dilute solution of the polymer poly (1,4-phenylene-2,6benzobisthiazole), $\mathrm{PBT}$, have shown it to have a rodlike conformation, with a persistence length $\rho$ comparable with or greater than the chain contour length $L$, and the polymer is known to be heavily protonated in the protic acids used to prepare its solutions. Despite the nacroion character of the chain in solution, it has been found that (probably metastable) aggregates can exist in which the rodlike molecules are associated in parallel arrays (ref. 1,3). In this study, copolymers of PBT,

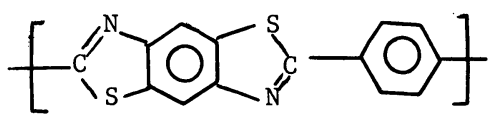

PBT

in which a small fraction of the phenylene residues are replaced by a bipyridyl unit are examined.

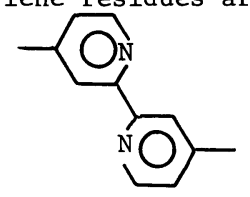

BIPYRIDYL UNIT

This unit introduces the possibility for rotational isomerizations that will reduce $\rho$, and makesthe root mean square radius of gyration $R_{G}$ much smaller than its value $L / \sqrt{12}$ for a rodlike chain. The copolymers will be designated by the notation PBT-co-m, where $\mathrm{m} / 100$ is the mole fraction of the phenylene residues replaced in the copolymer. As illustrated by the rotational conformer shown for the bipyridyl unit, a staggered rodlike conformer is available to this PBT-co-m, which may explain the appearance of nematic solutions with the copolymer at high enough concentration. In this study, polarized and depolarized light scattering was used to determine the Rayleigh ratios $R_{V v}(q)$ and $R_{H v}(q)$, respectively, and photon correlation light scattering was used to determine the intensity autocorrelation function $g(2)(\tau, q)$, all as a function of the polymer concentration $c$ and the scattering angle $\theta$, with $q=(4 \pi / \lambda)$ $\sin (\theta / 2)$. With polydispersed samples, these are given in terms of summations over the scattering entities (ref. 1,3-5).

$$
\begin{aligned}
& \frac{R_{V v}(0)}{K}=\sum_{i} M_{i}\left(1+\frac{4}{5} \delta_{i}^{2}\right) c_{i}-2 \sum \sum_{i j} M_{i} M_{j}\left(A_{2}\right)_{i j} c_{i} c_{j}+\ldots \\
& \left(\frac{R_{v v}(q)}{c}\right)^{0}=\left(\frac{1}{c} \sum_{i} R_{V v, i}(q)\right)^{0}
\end{aligned}
$$




$$
\begin{aligned}
& \left(\frac{R_{V v, i}(q)}{K c}\right)^{0}=c^{-1} M_{i} c_{i}\left(1+\frac{4}{5} \delta_{i}^{2}\right)\left\{1-\frac{1}{3} J_{i} R_{G, i}^{2} q^{2}+\ldots\right\} \\
& J_{i}^{-1}=\left[1-\frac{4}{5}\left(f_{1} \delta\right)_{i}+\frac{4}{7}\left(f_{2} \delta\right)_{i}^{2} j /\left(1+\frac{4}{5} \delta_{i}^{2}\right)\right. \\
& \frac{R_{H v}(q)}{K}=\frac{3}{5} \sum_{i} M_{i} c_{i} \delta_{i}^{2}\left[1-\frac{3}{7} f_{3, i} R_{G, i}^{2} q^{2}+\ldots\right] \\
& \left(\frac{g^{(2)}(\tau, q)-1}{{ }^{(2)}(0, q)-1}\right)^{0}=\left\{\sum_{i} r_{i}(q) \exp \left(-\gamma_{i} q^{2} \tau\right)\right\} 2 \\
& r_{i}(q)=\left(\frac{R_{V v, i}(q)}{R_{V v}(q)}\right)^{0}
\end{aligned}
$$

Here, $\mathrm{K}$ is an optical constant, superscript (0) indicates a quantity extrapolated to infinite dilution, $R_{G, i}$ is the root-mean square radius of gyration for component $i$, having molecular weight $M_{i}$ and concentration $c_{i}, \delta_{i}$ and the $\left(f_{j}\right)_{i}$ depend on the orientational correlation among the scattering elements in component $i,\left(A_{2}^{i}\right)_{i j}$ is an interaction coefficient, equal to the second virial coefficient for $i=j$, and $\gamma_{j}$ is a constant for each $i$ (for $R_{G, i} q$ less than about 3 ), conveniently expressed as a hydrodynamic radius $R_{H, i}$, with

$$
\mathrm{R}_{\mathrm{H}, \mathrm{i}}=\mathrm{kT} / 6 \pi \mathrm{n}_{\mathrm{s}} \gamma_{\mathrm{i}}
$$

Equations 1-5 are most frequently applied to describe the effects of the molecular weight distribution of fully dissolved solute. Here, these expressions will be used to characterize the properties of pseudo-components representing the fully dissolved species as one pseudo-component (with neglect of its molecular weight distribution), and the aggregates as additional pseudo-components. This procedure is a useful approximation provided the pseudocomponents (hereafter referred to simply as components) differ sufficiently in their properties (ref.3). Data on any nonexponential nature of $(g(2)(\tau, q))^{\frac{1}{2}}$ will be utilized to determine $r_{i}(q)$ and $R_{H}$ for the components. If the treatment is reasonable, it is expected that $\mathrm{R}_{\mathrm{H}}{ }_{i}$ will be $\mathrm{H}^{\mathrm{H}}$ independent of $q$, but that $\mathrm{r}_{i}(\mathrm{q})$ will vary significantly with $q$ as dictated by ${ }^{\mathrm{H}}$ Eqn. 2. The estimates for $\mathrm{r}_{i}(q)$ can subsequently be used in analysis of $\mathrm{R}_{\mathrm{Vv}}(\mathrm{q})$ to determine approximations to $\mathrm{R}_{\mathrm{Gi}}$.

The solutions to be considered will be solutions of PBT or PBT-co-m in methane sulfonic acid, MSA, or in MSA in which the potassium salt of the acid is dissolved. With the latter solvent, $A_{2}$ appears to decrease toward zero, and a substantial increase in $R_{H v}(q)$ is observed.

\section{EXPERIMENTAL}

The PBT-co-m copolymers were provided by Dr. R.C. Evers, Wright-Patterson Air Force Base, Ohio. They were received as dry powders prepared by precipitation from the polyphosphoric acid, PPA, polymerization solvent in water, followed by extensive washing in water to remove residual $\mathrm{P}_{2} \mathrm{O}_{5}$. The $\mathrm{PBT}$ polymers used here are described in detail in (ref. 1). The MSA was distilled under vacuum and stored as described in (ref.1-3). The potassium salt of MSA was prepared by titration of MSA with potassium hydroxide. The salt crystals were recrystallized from water and dried for use. Polymers were dried under vacuum prior to use. Solutions in MSA containing the salt were prepared in two ways with PBT-co-5: the polymer was dissolved in MSA containing the salt or the salt was dissolved in a solution of the polymer in MSA. Solutions of PBT were prepared by the latter method.

The light scattering methods used here are those described in (refs. 1 and 3). Solutions were prepared for light scattering by dilution of a stock solution with the appropriate solvent. Before dilution, the stock solution was subjected to sonication for 1 hour, followed by centrifugation (ca $5000 \mathrm{G}$ ) for $24 \mathrm{hrs}$ to reduce aggregation (ref. 3). The stock solution was then diluted and filtered through a 2-5 $\mu \mathrm{m}$ teflon membrane filter into light scattering cells ( $5 \mathrm{~m} 1$ volume). The cell was sealed under vacuum to prevent contanination by moisture, which is known to promote aggregation. Light scattering data were obtained directly on these solutions, as well as these solutions after an additional period of centrifugation (24 hr) using a swing-bucket rotor. In no case did this result in detectable removal of the solute.

Photon correlation data were obtained with $514.5 \mathrm{~nm}$ wavelength 1 ight. Fluorescence and absorption corrections to the observed intensities were made as in (ref. 1). In part, fluorescent contribution to the measured intensity was minimized by placing a narrow bandpass interference filter centered at the wavelength $\lambda_{0}$ of the incident beam in front of the photomultiplier. Fluorescence at this wavelength could affect estimates of the observed scattering (especially $R_{\text {Hy }}(q)$ ). The (relative) scattering $I_{+}$between crossed polars was routinely measured at $20 \mathrm{~nm}$ increments over the range from $50 \mathrm{~nm}$ below $\lambda_{0}$ to $700 \mathrm{~nm}$ using 
an interference filter with variable transmission along its length (Oriel, mode1 $57480 \quad$ ). In this way, true $\mathrm{R}_{\mathrm{Hv}}(\mathrm{q})$ scattering was readily apparent in comparison with fluorescence at $\lambda_{0}$. In addition, $\mathrm{Hv}$ the interpolated fluorescence at $\lambda_{0}$ and a convenient wavelength $\lambda_{1}$ about $30 \mathrm{~nm}$ larger than $\lambda_{0}$ could be determined. These data were subsequently used to deduce fluorescence corrections needed to compute $\mathrm{R}_{\mathrm{Vv}}(\mathrm{q})$ and $\mathrm{R}_{\mathrm{Hv}}(\mathrm{q})$ from experimental data on the observed intensities at $\lambda_{0}$ and $\lambda_{1}$.

Solution viscosities were measured with a suspended level, Cannon-Obbelohde viscometers, making sequential dilutions in the viscometer. The solution was maintained under dry $\mathrm{N}_{2}$ at all times. Data on the relative viscosity $n_{r e 1}=n / n_{s}$, with $n_{s}$ the solvent viscosity, were analyzed with the expressions $\left(n_{\mathrm{sp}}=n_{\mathrm{rel}}{ }^{-1}\right)$ :

$$
\begin{aligned}
& \frac{\eta_{s p}}{c}=[n]+k^{\prime}[n]^{2} c+\ldots \\
& \frac{1 n n_{r e 1}}{c}=[n]-\left(\frac{1}{2}-k^{\prime}\right)[n]^{2} c+\ldots \\
& {[n]_{c} \equiv c^{-1}\left[2\left(\eta_{s p}-1 n \eta_{r e 1}\right)\right]^{1 / 2}} \\
& {[n]_{c}=[n]-\left(\frac{1}{3}-k^{\prime}\right)[n]^{2} c+\ldots}
\end{aligned}
$$

Of these, $[n]_{c}$ is often found to be nearly independent of $c$ over the range of $c$ of interest inasmuch as $k^{i} \approx 1 / 3$.

\section{RESULTS}

Solutions in methane sulfonic acid

The viscometric results are conveniently given in terms of [n]. For most polymer solutions, $[n]_{c}$ is a close approximation to $[n]$. For some of the solutions studied here, $[n]_{c}$ exhiited dependence on $c$ that could be empirically represented by the empirical expression

$$
[n]_{c}^{-1}=[n]_{\mathrm{APP}}^{-1}+\mathrm{mc}^{1 / 2}
$$

Values of $[n]_{A P P}$ may most reliably be considered to represent $[n]$ when $m$ is zero. Examples. are known (ref. 2) for which $[n]_{c}$ could be fitted by Eqn. 9 for $[n]_{c}>0.05$, but for whicil deviation occurred for smaller $c$ such that $[n]_{\text {APP }}>[\eta]$. Nevertheless, with the solutions

\begin{tabular}{|c|c|c|c|c|}
\hline Polymer & $\begin{array}{l}\text { PBT-co-5 } \\
\text { No salt }\end{array}$ & $\begin{array}{l}\text { PBT-co-5 } \\
\text { with salt }\end{array}$ & $\begin{array}{l}\text { PBT }-72^{b} \\
\text { No salt }\end{array}$ & $\begin{array}{l}\text { PBT-72 } \\
\text { with salt }\end{array}$ \\
\hline $\begin{array}{l}\mathrm{X} \\
\rho / \mathrm{nm}\end{array}$ & $\begin{array}{l}0.05 \\
19\end{array}$ & $\begin{array}{l}0.05 \\
19\end{array}$ & $0_{--}$ & 0 \\
\hline $\begin{array}{l}{[n] / \mathrm{mlg}^{-1}} \\
{[n]^{\frac{1}{2}} \partial[n]_{\mathrm{c}}^{-1} / \partial c^{\frac{1}{2}}}\end{array}$ & $\begin{array}{l}1,250 \\
0.92\end{array}$ & $\begin{array}{l}610 \\
0\end{array}$ & $\begin{array}{l}1,620 \\
0\end{array}$ & $\begin{array}{l}970 \\
-0.47\end{array}$ \\
\hline $\begin{array}{l}\left(\mathrm{R}_{\mathrm{Yv}}(0) / \mathrm{Kc}\right)^{0} \\
\left\{\partial \ln \left(\mathrm{c} / \mathrm{R}_{\mathrm{Vv}}(0)\right)^{\frac{1}{2}} / \partial \mathrm{c}\right\}^{0} / \mathrm{m}^{-1} \mathrm{~g}^{-1} \\
\left\{3\left[\partial \ln \left(\mathrm{c} / \mathrm{R}_{\mathrm{Vv}}(0)\right) / \partial \mathrm{q}^{2}\right]^{0}\right\} / \mathrm{nm}\end{array}$ & $\begin{array}{l}100,000 \\
1,500 \\
40\end{array}$ & $\begin{array}{l}250,000 \\
0 \\
50-100\end{array}$ & $\begin{array}{l}34,000 \\
1,030 \\
43\end{array}$ & $\begin{array}{l}1,500,000 \\
2,100 \\
315\end{array}$ \\
\hline $\begin{array}{l}(5 / 3)\left(\mathrm{R}_{\mathrm{Hv}}(0) / \mathrm{Kc}\right)^{0} \\
\left\{\partial \ln \left(\mathrm{c} / \mathrm{R}_{\mathrm{Hv}}(0)\right)^{\frac{1}{2}} / \partial \mathrm{c}\right\}^{0} / \mathrm{mlg}^{-1} \\
{\left[(7 / 3)\left(\partial \ln \left(\mathrm{c} / \mathrm{R}_{\mathrm{Hv}}(0)\right) / \partial \mathrm{q}^{2}\right)^{0}\right] / \mathrm{nm}}\end{array}$ & $\begin{array}{l}<100 \\
-- \\
--\end{array}$ & $\begin{array}{l}6,500 \\
-400 \\
30-50\end{array}$ & $\begin{array}{l}3,300 \\
0 \\
24\end{array}$ & $\begin{array}{l}33,000 \\
0 \\
60\end{array}$ \\
\hline $\begin{array}{l}\mathrm{R}_{\mathrm{H}, 1} / \mathrm{nm} \\
\mathrm{R}_{\mathrm{H}, 2} / \mathrm{nm} \\
\mathrm{R}_{\mathrm{H}, 3} / \mathrm{nm}\end{array}$ & $\begin{array}{l}3-5 \\
17-30 \\
--\end{array}$ & $\begin{array}{l}4 \\
9-14 \\
110-180\end{array}$ & $\begin{array}{l}13 \\
--\end{array}$ & $\begin{array}{l}5.3 \\
40 \\
180\end{array}$ \\
\hline
\end{tabular}
studied here, Eqn. 9 appears to apply to $c$ as low as could be studied, so we will consider that $[n]_{\text {APP }}$ is a reasonable approximation to $[\eta]$. Values of $[n] \approx[n]_{\text {APP }}$ and $\partial[\eta] c_{c}^{-1 / \partial c^{\frac{1}{2}}}$ are listed in Table 1.

TABLE 1. Light scattering and viscometric data on solutions in methane sulfonic acid

a) $0.45 \mathrm{~N}$ for $[\eta] ; 0.56 \mathrm{~N}$ for the light scattering data b) Data from reference 1

c) $1.0 \mathrm{~N}$ for $[n] ; 0.3 \mathrm{~N}$ for the light scattering data 
For the solutions of PBT-co-5, $\mathrm{R}_{\mathrm{Hv}}(\mathrm{q})$ is negligibly small. Thus, for the data shown in Fig. 1 , the relative intensity $I_{+}$observed between crossed polars over the wavelength 480 to 700 $\mathrm{nm}$ with $514 \mathrm{~nm}$ wavelength incident light is smaller, and not larger than the fluorescence excitation at $514 \mathrm{~nm}$. Centrifugation of these solutions produced only modest effect on $\mathrm{R}_{\mathrm{Vv}}(0)$ as shown in Fig. 2. Data on $\mathrm{g}(2)(\tau, q)$ were found to be nonexponential for solutions of PBT-co-5, and could be analyzed with Eqn. 4. This effect is pronounced for the solutions prior to centrifugation, giving rise to components with $R_{H}, i$ equal to $3-5$ and $17-30 \mathrm{~nm}$, see Table 1 ; in calculation of $R_{\mathrm{H}}$ with Eqn. $5, \mathrm{n}_{\mathrm{s}} / \mathrm{Pa} \cdot \mathrm{s}$ for MSA is equal to $8.64 \times 10^{-7} \exp (2800 /$ T). Data on $r_{i}(q)$ and $R_{H}$, for solutions of PBT-co-5 in MSA are given in Fig. 3. The component with $R_{H_{i}}=17-30 \mathrm{~nm}$ dominates the data on $R_{V v}(q)$. Parameters deduced from $c / R_{V v}(q)$ are listed in Table 1.

Fig. 1 The relative depolarized intensity $\mathrm{I}_{+}$as a function of the wavelength $\lambda$ for $514.5 \mathrm{~nm}$ wavelength excitation: -- $0.51 \mathrm{~g} / 1$ in MSA, and in MSA with $0.56 \mathrm{~N}$ of the potassium salt of MSA.
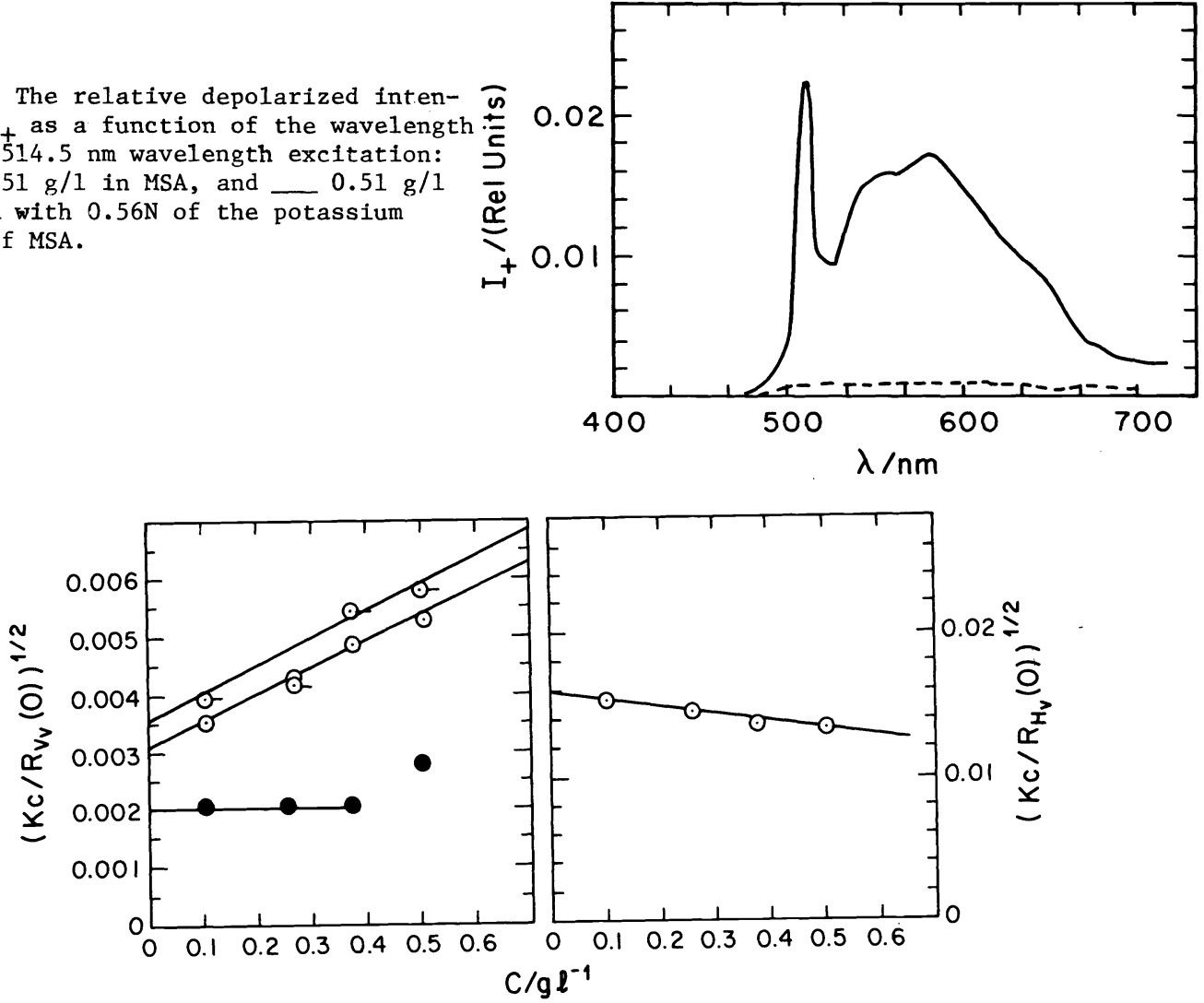

Fig. 2. Light scattering data on solutions of PBT-co-5. Right: $\left(\mathrm{Kc} / \mathrm{R}_{\mathrm{Vv}}(0)\right)^{\frac{1}{2}}$ vs $c$ for solutions in MSA, 0 , filtered solution, $Q$, centrifuged sample, and in MSA containing $0.56 \mathrm{~N}$ of the potassium salt of $\mathrm{MSA}, \bullet$; Left: $\left(\mathrm{Kc}_{\mathrm{c}} / \mathrm{R}_{\mathrm{Hv}}(0)\right)^{\frac{1}{2}}$ vs $\mathrm{c}$ for solutions in MSA containing $0.56 \mathrm{~N}$ salt as above.

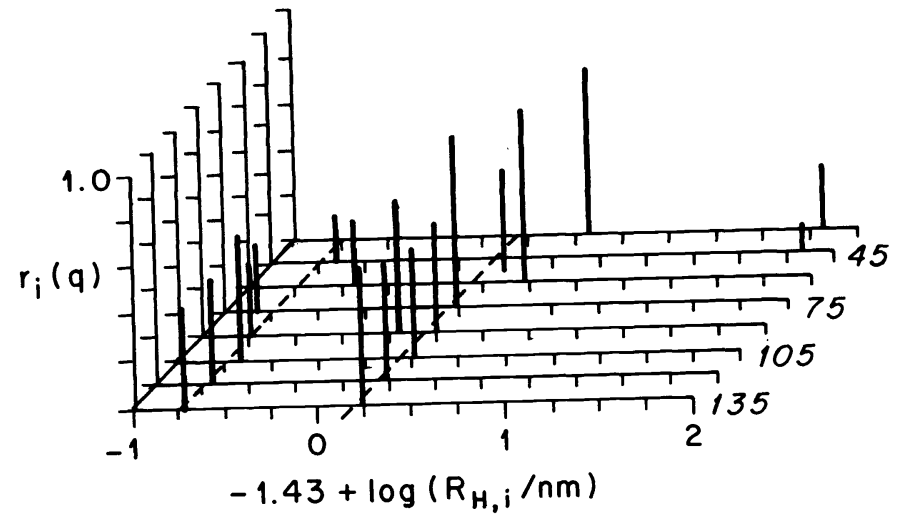

Fig. 3. Representative data for $R_{H, i}$ and $r_{i}$ (q) for the scattering angles $30 \leq \theta \leq$ 135 as labeled. The data are for ${ }_{\mathrm{PBT}-\mathrm{co}-5}$ in MSA with $\mathrm{c}=0.25 \mathrm{~g} / 1$. 
Solutions in methane sulfonic acid containing salt As above, the viscometric data are presented in terms of $[n]_{c}$. Estimates of $[n] \tilde{n}[n] \mathrm{APP}$
and $\partial[n]^{-1} / \partial c^{\frac{1}{2}}$ obtained with Eqn. 9 are entered in Table 1 for solutions containing the potassium salt of MSA.

As shown in Fig. 1, addition of salt to solution of PBT-co-5 in MSA results in a marked increase in the fluorescence $\mathrm{I}_{+}$over a wide range in wavelength, along with a clear marked increase in $\mathrm{R}_{\mathrm{Hv}}(\mathrm{q})$. The results for $\mathrm{Kc} / \mathrm{R}_{\mathrm{Hv}}(0)$ and $\mathrm{Kc} / \mathrm{R}_{\mathrm{Vv}}(0)$ are given in Fig. 2, and parameters entracted from the data on $\mathrm{Kc} / \mathrm{R}_{\mathrm{Vv}}(\mathrm{q})$ and $\mathrm{Kc} / \mathrm{R}_{\mathrm{Hv}}(\mathrm{q})$ are 1isted in Table 1 . Since the effects observed are about the same for solutions prepared by dissolving the polymer in MSA containing salt, or by dissolving the salt in MSA containing the polymer, these are not distinguished here. Analysis of $g(2)(\tau, q)$ for solutions of PBT-co-5 with Eqns. 4-5 results in three components. Values of $R_{H}$, are given in Table 1 ; in calculating $R_{H}$, from $\gamma_{i}$, $\eta_{s}$ is computed from $\partial\left(n_{\mathrm{s}} / \mathrm{mPa} \cdot \mathrm{s}\right) / \partial\left(\mathrm{m} / \mathrm{mol} 1^{-1}\right)=0.142$, with m the concentration of the salt. Use of the corresponding data on $r_{i}(q)$ and Eqn. 2 provide estimates for $\left(R_{G}^{2} / M\right)_{i}$. For PBT-co-5, over most of the scattering angles, the scattering is dominated by the component with $R_{H, i}=$ 9-14 nm. Consequently, the estimate of $\left[3\left(R_{V v}(0) / c\right)^{0}\left(\partial\left(c / R_{V v}(q)\right) / \partial q^{2}\right)^{0}\right]^{\frac{1}{2}}$ for this component is similar to that obtained for the total scattering if the analysis is restricted to scattering angles above $60 \mathrm{deg}$. , namely about $100 \mathrm{~nm}$. Qualitatively, similar results were obtained for solutions containing 1 and $1.5 \mathrm{~N}$ salt.

With PBT, the solution in MSA exhibits $\mathrm{R}_{\mathrm{HV}}(\mathrm{q})$ scattering appropriate to the molecular anisotropy $\delta$ of the rodlike chain (ref. 1). Addition of the potassium salt results in 5 to $10-$ fold increase in $\mathrm{R}_{\mathrm{Hv}}(0)$, as shown in Fig. 4. Similarly, $\mathrm{R}_{\mathrm{Vv}}(0)$ is increased appreciably, see Fig. 4, and $g(2)(\tau, q)$, which is essentially an exponential function for the solution in MSA, is markedly nonexponential with the addition of salt. Values of $R_{H, i}$ are entered in Table 1. The component with largest $R_{H, i}$ dominates $R_{V v}(0)$, although this component is not necessarily present in large concentration.

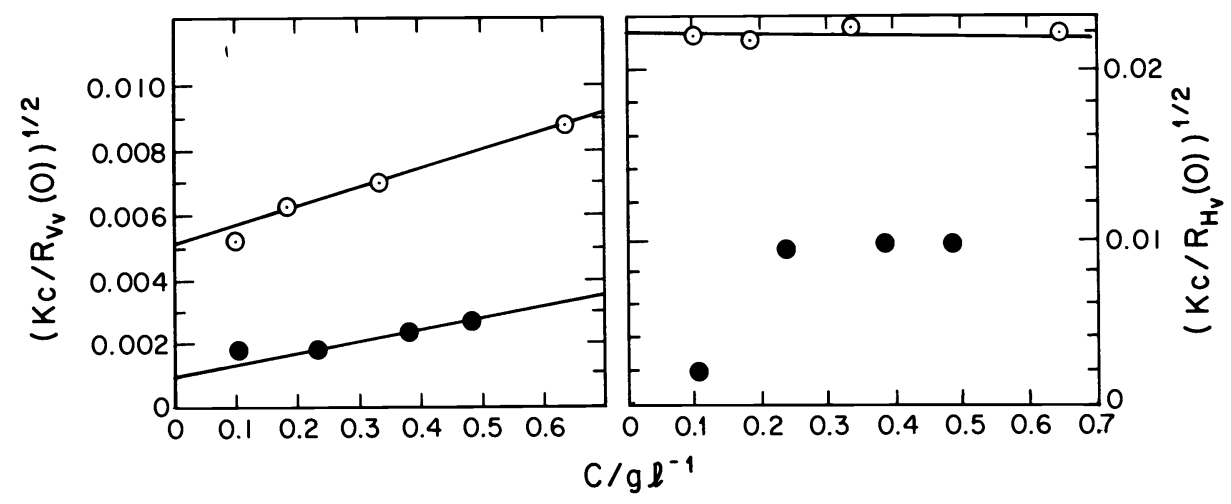

Fig. 4. Light scattering data for PBT-72. Right: $\left(\mathrm{Kc} / \mathrm{R}_{\mathrm{VV}}(0)\right)^{\frac{1}{2}} \underline{\mathrm{vs}} \mathrm{c}$ for solutions in MSA, 0 , and in MSA containing $0.3 \mathrm{~N}$ of the potassium salt of MSA, Left: $\left(\mathrm{Kc} / \mathrm{R}_{\mathrm{Hv}}(0)\right)^{\frac{1}{2}}$ vs $\mathrm{c}$ with the symbols identified as above.

Solutions in MSA

The depolarized scattering is given in terms of the optical anisotropy $\delta$ by the expression

$$
\begin{aligned}
& \mathrm{R}_{\mathrm{Hv}}(0)=\frac{3}{5} \mathrm{Kc} \mathrm{M}_{\mathrm{w}} \delta^{2} \\
& \delta^{2}=\delta_{0}^{2}\left(\frac{1}{n}\right)^{2} \sum_{i j} \sum_{j} \frac{3<\cos ^{2} \gamma_{i j}>-1}{2}
\end{aligned}
$$


with the sums over all of the rodlike chain segments, where $\gamma_{i j}$ is the angle between the optical axes of segments $i$ and $j$, each of which has an intrinsic optical anisotropy $\delta_{0}$ $\left(\delta_{0}=0.5\right.$ for $\left.\mathrm{PBT}\right)$. For the copolymers studied here, in the extreme case with no rotational restriction at the bipyridyl unit Eqn. 10 becomes

$$
\mathrm{R}_{\mathrm{Hv}}(0)=\frac{3}{5} \mathrm{Kc}\left(\mathrm{M}_{\mathrm{R}}\right)_{\mathrm{w}} \delta_{0}^{2}
$$

where $M_{R}$ is the molecular weight of the rodlike PBT chain sequence in the copolymer. If the fraction $(1-x)>0$ of the phenylene moieties replaced by bipyridyl units are randomly distributed, then $\left(M_{R}\right)_{w} \approx m_{0}(2-x) / x$, where $\mathrm{m}_{0}$ is the PBT repeat unit molecular weight $\left(m_{0}=266\right)$. With $\delta_{0}=0.5$, it is seen that $R_{H y}(0)$ is small for the value of $x$ of interest here. An improved estimate for $\mathrm{R}_{\mathrm{Hv}}(0)$ can be made using Eqn. 11 for $\delta$ provided the orientational distribution among the segments is known. For the wormlike chain mode1, which should be reasonable for the fully dissolved copolymers, Eqn. 11 gives (ref. 4,6-8)

$$
\delta^{2}=\delta_{0}^{2}(2 \rho / 3 L)\{1-(\rho / 3 L)[1-\exp (-3 L / \rho)]\}
$$

where $\rho$ is the persistence length, $L$ the chain contour length, $\delta_{0}$ the intrinsic anisotropy, and $\ell$ the length of a chain element with molecular weight $\mathrm{m}_{0}$. For the wormlike chain (ref. 4)

$$
\begin{aligned}
& R_{G}^{2}=\frac{L \rho}{3} S(\rho / L) \\
& S(y)=1-3 y+6 y^{2}-6 y^{3}\left[1-\exp \left(-y^{-1}\right)\right] \\
& S(y) \approx(1+4 y)^{-1}
\end{aligned}
$$

For a polydispersed polymer with a Schulz-Zimm distribution of $M$, the light scattering averaged mean square radius of gyration is given by

$$
\begin{aligned}
& \mathrm{R}_{\mathrm{G}, \mathrm{LS}}^{2}=\frac{\mathrm{L}_{\mathrm{z}} \rho}{3} \overline{\mathrm{S}}\left(\rho / \mathrm{L}_{\mathrm{z}}\right) \\
& \overline{\mathrm{S}}(\mathrm{y})=1-3 \mathrm{y}+6 \mathrm{y}^{2} \frac{\mathrm{h}+2}{\mathrm{~h}+1}-6 \mathrm{y}^{3} \frac{(\mathrm{h}+2)^{2}}{\mathrm{~h}(\mathrm{~h}+1)}\left\{1-\left[1+\frac{1}{\mathrm{y}(\mathrm{h}+2)}\right]^{-h}\right\} \\
& \overline{\mathrm{S}}(\mathrm{y}) \approx[1+4 \mathrm{y}(\mathrm{h}+2) /(\mathrm{h}+3)]^{-1}
\end{aligned}
$$

where $1+h^{-1}=M_{w} / M_{n}$ and $L_{z}=M_{z} / M_{L}=(h+2) M_{w} /(h+1) M_{L}$; with $M_{L}$ the mass per unit length. Using Eqn. $15 \mathrm{data}$ on $\partial\left[\mathrm{Kc} / \mathrm{R}_{\mathrm{Vy}}(\mathrm{q})\right] / \partial \mathrm{q}^{2}$ provide an estimate for $\rho[(\mathrm{h}+2) /(\mathrm{h}+1)] \overline{\mathrm{s}}\left(\rho / \mathrm{L}_{\mathrm{z}}\right)$. With the copolymers of interest here. $R_{G}^{2}$ must also be averaged to account for the distribution of the chain lengths $\ell_{1}$ between, ${ }^{2}$ the bipyridyl moieties. If the latter is independent of $\mathrm{L}$, then for small $\rho / \mathrm{L}, \overline{\mathrm{S}}\left(\rho / \mathrm{L}_{\mathrm{z}}\right) \approx 1$ and a simple arithmetic average over the distribution of $\rho$ is appropriate. Thus, with Eqn. 15,

$$
\vec{s}\left(\rho / L_{z}\right)=9 M_{L}\left(\frac{h+1}{h+2}\right) \lim _{q=0} \frac{\partial\left[K c / R_{V v}(q)\right]^{0}}{\partial q^{2}}
$$

Calculations of $\mathrm{R}_{\mathrm{H}}$ for the wormlike chain models (ref. 9) can be represented by the relation

$$
\mathrm{R}_{\mathrm{H}}=\mathrm{L} / 2\left[(27 \mathrm{~L} / 16 \rho)^{\varepsilon / 2}+1 \mathrm{n}^{\varepsilon}(3 \mathrm{~L} / 2 \mathrm{a})\right]^{-1 / \varepsilon}
$$

where $a$ is the chain diameter and $\varepsilon=2$, so that for small $\rho / L, R_{H} \approx 2 R_{G} / 3$, as expected for a flexible chain polymer.

An estimate for $\rho$ for the copolymers of interest here may be obtained by representing the chain as a sequence of rodlike elements of length $\ell_{1}=M_{R} / M_{L}$ alternating with elements of length $\ell_{2}$ equal to the distance between the centers of adjacent pyridyl rings, with a fixed angle $\alpha$ between successive elements. Then, (ref. 10)

$$
2 p=\frac{\ell_{1}^{2}+l_{2}^{2}}{\ell_{1}+l_{2}}\left\{\frac{1+q}{1-q}-\frac{2\left(\ell_{1}-l_{2}\right)^{2}}{\ell_{1}^{2}+l_{2}^{2}}\left(q+q^{3}\right)\right\}
$$

where $\mathrm{q}=\cos \alpha$. Since $\ell_{1} \gg \ell_{2} \approx 0.44 \mathrm{~nm}, \ell_{1} \approx\left(\mathrm{M}_{\mathrm{R}}\right)_{\mathrm{n}} / \mathrm{M}_{\mathrm{L}} \approx \ell / \mathrm{x}$ if random placement of the bipyridyl units is assumed, where $\ell=\mathrm{m}_{\mathrm{O}} / \mathrm{M}_{\mathrm{L}}$, and the factor in brackets is about equal to 1.54 .

The $\mathrm{M}_{\mathrm{w}}$ obtained for PBT-co-5 is unexpectedly large for a step-growth polymer. Moreover, analysis of $g^{(2)}(\tau, q)$ with Eqn. 4 reveals two (pseudo) components, with the major 
contribution to $\mathrm{g}^{(2)}(\tau, \mathrm{q})$ having $\mathrm{R}_{\mathrm{u}} \approx 17-30 \mathrm{~nm}$ over the range of $\mathrm{c}$ studied, with a minor contribution having $\mathrm{R}_{\mathrm{H}} \approx 3-5 \mathrm{~nm}$. The latter is attributed to low molecular weight rodlike chains with contour length $\mathrm{L}_{\mathrm{z}} \approx 15 \mathrm{~nm}$ that is smaller than the length $\left(\mathrm{M}_{\mathrm{R}}\right)_{\mathrm{z}} / \mathrm{M}_{\mathrm{L}} \approx 70 \mathrm{~nm}$ expected between bipyridyl units with PBT-co-5. The component with $\mathrm{R}_{\mathrm{H}} \tilde{\sim} \mathrm{R}_{1}{ }_{-30} \mathrm{~nm}$ is essentially the only contribution to $\mathrm{R}_{\mathrm{Y}}(\mathrm{q})$. The estimate $\rho \overline{\mathrm{S}}\left(\rho / \mathrm{L}_{z}\right)$ estimated with the latter using Eqn. 16 is smaller than expected (e.g. $11 \mathrm{~nm}$ even if the factor $(h+1) /(h+2) \approx 1)$. Moreover, using Eqn. 17, the observed $R_{H}$ correspond to calculated $M_{z}$ smaller than the observed $M_{w}$. These disparities are attributed to aggregation, which results in a supramolecular structure containing several chains. The viscometric data are in accord with this conjecture. Thus, in general,

$$
[n]=\mathrm{K}_{\pi} \mathrm{N}_{\mathrm{A}} \cdot \mathrm{R}_{\mathrm{G}}^{2} \mathrm{R}_{\mathrm{H}} \mathrm{M}^{-1}
$$

with $\mathrm{K}=1$ for rodlike chains and $\mathrm{K}=3$ for flexible chain polymers with large M. With the estimate $\mathrm{R}_{\mathrm{H}} \approx 20 \mathrm{~nm}$ and $\mathrm{R}_{\mathrm{G}}^{2} / \mathrm{M}_{\mathrm{w}}=3 \partial\left[\mathrm{Kc} / \mathrm{R}_{\mathrm{Vv}}(\mathrm{q})\right] / \partial \mathrm{q}^{2} \approx 0.016 \mathrm{~nm}^{2} \mathrm{~d}^{-1}$, use of Eqn. 19 gives [n] $\approx 1800 \mathrm{mlg}^{-1}$, in comparison with the observed value of $1250 \mathrm{mlg}^{-1}$ obtained with Eqns. 8 and 9 in the limit of infinite distribution, whereas [n] decreases with increasing c(e.g., $\left.\partial[n]^{-1} / \partial c^{\frac{1}{2}} \approx 0.026 \mathrm{ml}^{\frac{1}{2}} \mathrm{~g}^{-\frac{1}{2}}\right)$. The supramolecular structure is considered to have a configuration similar to that for a randomly branched polymer. Hence, Eqn. 19 provides a reasonable estimate for $[n]$, and $R_{H} \approx 2 R_{G} / 3$. With such a structure, for a strong excluded volume effect, it is reasonable to use the approximation $\mathrm{A}_{2} \mathrm{M}_{\mathrm{w}} \approx[n]_{\mathrm{c}}$ at each $\mathrm{c}$, so that $\mathrm{R}_{\mathrm{Vv}}(0)$ can be expressed in the form

$$
\frac{\mathrm{Kc}}{\mathrm{R}_{\mathrm{Vv}}(0)}=\left(\mathrm{M}_{\mathrm{w}, 0} \nu_{\mathrm{w}}\right)^{-1}\left(1+[\eta]_{c}\right)^{2}
$$

where $\mathrm{M}_{\mathrm{w}, 0}$ is the true molecular weight, and $\nu_{\mathrm{w}}$ is the degree of association, dependence on c. With Eqn. $20, \mathrm{M}_{\mathrm{w}}, \mathrm{\nu}_{\mathrm{w}}$ increases two-fold over the range of c studied, with $\mathrm{M}_{\mathrm{w}}, 0_{\mathrm{w}} \approx$ $10\left(M_{R}\right)_{W}$ at infinite, dilution, where $\left(M_{R}\right)_{W}$ is the calculated molecular weight of the rodlike chain sequences.

\section{Solutions in MSA containing salt}

The substantial changes in $\mathrm{R}_{\mathrm{Vy}}(\mathrm{q})$ and $\mathrm{R}_{\mathrm{Hv}}(\mathrm{q})$ caused by the addition of the potassium salt of MSA are attributed to enhanced intermolecular orientational and spatial order. With PBT, this effect results in a large increase in $\nu_{w}$, with considerable orientational orientation among the rodlike chains in the aggregates. Thus, data on $R_{\mathrm{Vv}}(0)$ and $\mathrm{R}_{\mathrm{Hv}}(0)$ gives $\nu_{\mathrm{w}}$ from (see Eqns. 1-3)

$$
\left(M_{D}\right)_{w} \nu_{w}=\left(\frac{R_{V v}(0)}{K c}-(4 / 3) \frac{R_{H v}(0)}{K c}\right)^{0}
$$

where $M_{D}$ the molecular weight of the rodlike chain. Comparison of the data on solutions without salt $\left(\nu_{\mathrm{w}} \approx 1\right)$ with data for the solution containing salt gives $\nu_{\mathrm{w}} \approx 50$ for the latter. With the expression

$$
\mathrm{R}_{\mathrm{Hv}}(0)=\frac{3}{5} \mathrm{Kc}\left(\mathrm{M}_{\mathrm{D}}\right)_{\mathrm{w}} \nu_{\mathrm{w}} \delta_{0}^{2} \mathrm{~g}_{2}
$$

the data on $\left(\mathrm{R}_{\mathrm{Hv}}(0) / \mathrm{c}\right)^{0}$ give $\mathrm{g}_{2}=0.2$ for the orientational correlation among the rodlike chains in the aggregates. Lacking a model for the supramolecular structure, $g_{2}$ cannot be directly interpreted, but it is assumed that $\left\langle\cos ^{2} \theta\right\rangle$ is common for all chains, where $\theta$ the angle between the axes of two chains, with the average carried out over all such pairs, then

$$
\mathrm{g}_{2}=\frac{3<\cos ^{2} \theta>-1}{2}
$$

and the results indicate a tendency for the chains to tend towards a parallel array in the aggregate.

The data on $\mathrm{R}_{\mathrm{Vv}}(0) / \mathrm{c}$ for the three larger concentrations give $\partial \ln \left(\mathrm{c} / \mathrm{R}_{\mathrm{Vv}}(0)\right) / \partial \mathrm{c} \approx 2100 \mathrm{~m} 1$, which is larger than the corresponding value obtained (ref. 1) in MSA (e.g., Table 1). In addition, the data give very large $R_{V v}(0) / c$ for the smallest $c$ and $[n]_{c}$ decreases with increasing $c$. The latter suggest that the state of aggregation, or the structure of the aggregates change with $c$, making detailed interpretation of the dependence on $c$ hazardous.

The data on $g^{(2)}(\tau, q)$ for the PBT solution containing salt exhibits a component with $\mathrm{R}_{H} \approx$ $40 \mathrm{~nm}$ that dominates the scattering at most angles. Additional components with $\mathrm{R}_{\mathrm{H}} \approx 5-7 \mathrm{~nm}$ and very large $R_{H}$ (variable with $c$, in the range 400 to $4000 \mathrm{~nm}$ ) are also observed. Application of Eqns. 1- $1-\frac{1}{4}$ to the data on $R_{V v}(q)$ results in an estimate of $40 \mathrm{~nm}$ for $\mathrm{J}^{-\frac{1}{2}} \mathrm{R}_{\mathrm{G}}$ for the component with $\mathrm{R}_{\mathrm{H}} \approx 40 \mathrm{~nm}$. The latter is similar to the data reported (ref. 1) for the polymer in the solution in MSA, but $R_{H}$ is larger than the value of $13 \mathrm{~nm}$ reported for the MSA solution, suggesting that this component may be sheath like in its overall shape, with a large number of the rodlike molecules packed in a more-or-less parallel array. Since the 
data do not provide a relative concentration of the components, an estimate for the molecular weight of the component, and hence its degree of association is not directly available. The components with large $R_{H}$ also exhibit values for $b_{V v}^{2}=-3 \ln \left[R_{V v}(q) r_{i}(q)\right] / q^{2}$ for small $q$ that are large and variable with $c\left(e . g ., b_{\mathrm{Vv}} 200-250 \mathrm{~nm}\right)$. The data on $\mathrm{b}_{\mathrm{Hy}}^{2}=-(7 / 3) 1 \mathrm{n}$ $\left[\mathrm{R}_{\mathrm{Hv}}(\mathrm{q})\right] / \mathrm{q}^{2}$ give a large correlation length, e.g., $\mathrm{b}_{\mathrm{Hv}} 30 \mathrm{~nm}$. As shown In Fig., the data at the lowest $c$ studied show more intense $R_{V v}(0)$ scattering than that for the larger $c$, with even larger $b_{\mathrm{Hv}}$. Since arrangement of the rodlike chains in the supramolecular structure is unknown, $f_{3}\left(e . g\right.$. Eqn. 3 ) is not known and $R_{G}$ cannot be computed from $b_{H v}$. Nevertheless, it is evident that the solution contains large aggregates with the chain with their axes nearly parallel.

Similar effects are observed with PBT-co-5 in that $\mathrm{R}_{\mathrm{Hv}}(\mathrm{q})$ is increased substantially on the addition of salt, with an accompanying increase in $\left(\mathrm{R}_{\mathrm{Vv}}(0) / \mathrm{c}\right){ }^{0}$. In addition, $\partial\left[\mathrm{Kc} / \mathrm{R}_{\mathrm{Vv}}(\mathrm{q})\right] /$ $\partial \mathrm{q}^{2}$ is not independent of $\mathrm{c}$ and a component with $\mathrm{R}_{\mathrm{H}} \approx \tilde{\tilde{0}} 110-180 \mathrm{~nm}$ appears in the contribution to $g(2)(\tau, q)$. The modest increase in $\left(R_{V v}(0) c\right) 0$ obtained on the addition of salt shows that relatively little additional association in comparison with the MSA solution. Rather, it appears that the principal effect is to increase the orientational correlation among the chains in the aggregates, e.g., the increased $R_{H v}(q)$. The negligible variation of [n]c with $c$ indicates that these aggregates are (meta) stable toward dilution. In that case, the negligible dependence of $c / R_{V v}(0)$ on $c$ indicates that $A_{2} \approx 0$. The similar value of $R_{H, i}$ and for the major component in MSA and one of the major components in the salt solution shows that the overall shape of some of the aggregates is not much altered by the addition of salt --the components with larger $\mathrm{R}_{\mathrm{H}}$ i observed with the salt solution may be caused by aggregation of the smaller aggregate steructure.

Theoretical studies on the phase equilibria (ref. 10,11) of mesogenic polymer solutions suggest that if $A_{2}$ is small enough and $c$ large enough, the mesogenic solutions will separate into two phases, a disordered solution with very small c, and an ordered solution with large c. Such effects have been reported with solutions of poly(benyl-L-glutamate) for concentrated solutions (ref. 13). This phase separation could be promoted by the creation of supramolecular aggregates, which effectively increases the local concentration $c_{10 c}$ for the polymer in the aggregates. It is possible that the effect on $R_{H v}(q)$, etc. observed on the addition of salt to MSA solutions is caused by the predicted phase separation for small $\mathrm{A}_{2}$, with the effect being enhanced here, because $c_{10 c}>c$. Similar considerations may be responsible for the marked tendency of rodlike chains to exhibit aggregation since the dissolution process requires passage through a concentrated solution, which may be unfavorable unless the effective polymer interactions are strongly repulsive (i.e. unless $\mathrm{A}_{2}$ is large and positive).

\section{Acknowledgement}

It is a pleasure to acknowledge partial support for this study from the Polymers Program, Division of Materials Research, National Science Foundation, and the Materials Program, Wright-Patterson Air Force Base.

\section{REFERENCES}

1. C. Lee, S-G. Chu and G.C. Berry, J. Polym. Sci., Polym. Phys. Ed. 21

2. P. Metzger Cotts and G.C. Berry, J. Polym. Sci., Polym. Phys. Ed. 21,1255 (1983).

3. Y. Einaga and G.C. Berry, in Microdomains in Polymer Solutions, ed. P. Dubin, in press.

4. H. Yamakawa, Modern Theory of Polymer Solutions, Harper and Row, New York (1971).

5. J.M. Schurr, CRC Crit. Rev. Biochem. 4, 371 (1977).

6. G.C. Berry, J. Polym. Sci., Polym. Symp. 65, 143 (1978).

7. F. Nagai, Polym. J. 3,67 (1972).

8. S.A. Allison, Biopolymers 22, 1545 (1983).

9. H. Yamakawa and M. Fujii, Macromolecules 6, 407 (1973).

10. M.V. Volkenstein, Configurationa1 Statistics of Polymer Chains, Wiley-Interscience, New York (1965).

11. R.A. Ronca and P.J. Flory, Mo1. Cryst. Liq. Cryst. 54, 289 (1979).

12. R.R. Matheson, Jr. and P.J. Flory, Macromolecules 14,954 (1981).

13. K. Tohyana and W.G. Miller, Nature (London) $289,8 \overline{13}$ (1981). 